Pacific Journal of Mathematics

CONTINUOUS LINEAR FUNCTIONALS ON CERTAIN

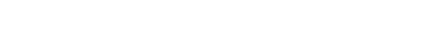




\section{CONTINUOUS LINEAR FUNCTIONALS ON CERTAIN TOPOLOGICAL VECTOR SPACES}

\section{S. CATER}

Let $X$ be a Lebesgue measurable subset of the real line and let $L$ be the set of all real valued measurable functions on $X$, two functions being identified if they are equal almost everywhere. S. Banach and M. M. Day have provided the following real topological vector spaces which have no nonzero continuous linear functionals (see [1] and [3]).

EXAMPLE OF BANACH. Let $V$ consist of all functions $f$ in $L$ for which $\int|f|(1+|f|)^{-1}<\infty$ and define the distance between two functions $f$ and $g$ in $V$ to be $\int|f-g|(1+|f-g|)^{-1}$. Then this distance function is a metric on $V$, and under the metric topology $V$ is a real topological vector space on which there is no nonzero continuous linear functional.

EXAMPLE OF DAY. Let $p$ be a real number for which $0<p<1$ and let $V$ consist of all functions $f$ in $L$ for which $\int|f|^{p}<\infty$. Define the distance between two functions $f$ and $g$ in $V$ to be $\int|f-g|^{p}$. Then this distance function is a metric on $V$, and under the metric topology $V$ is a real topological vector space on which there is no nonzero continuous linear functional.

On the other hand if $p \geqq 1$ in Day's problem then $\left(\int|f|^{p}\right)^{1 / p}$ is a norm on $V$ which gives rise to the well known Banach space $L_{p}(X, m)$, and of course a Banach space has nonzero continuous linear functionals by the Hahn-Banach Theorem. Observe that the Banach space $L_{p}(X, m)$ and the examples of Banach and Day have this in common; each is a real topological vector space, $V$, for which there is a nondecreasing function $\phi$ mapping the nonnegative real axis into itself such that a complete neighborhood system of $\theta$ in $V$ (where $\theta$ denotes the zero function) is given by sets of the form $\left\{f \in V ; \int \phi(|f|)<\varepsilon\right\}$. The purpose of the present paper is to provide a theorem on abstract measure spaces which unifies all these results and reveals other topological vector spaces on which there exists no nonzero continuous linear functional.

Let $(X, m)$ be a measure space as defined by Halmos in $[5$, p. 73$]$

Received November 16, 1962. 
and let $L$ be the set of all measurable functions on $X$, two functions being identified if they are equal almost everywhere. By a simple function on $X$ we mean a linear combination of characteristic functions of subsets of $X$ having finite measure. By an atom we mean a subset $U$ of finite positive measure such that given any measurable subset $E$ of $U$, either $m(E)=0$ or $m(E)=m(U)$. The atoms $U_{1}$ and $U_{2}$ are identified if $m\left(U_{1} \triangle U_{2}\right)=0$; equivalently, the atoms $U_{1}$ and $U_{2}$ are regarded as the same if $m\left(U_{1} \cap U_{2}\right)=m\left(U_{1}\right)$ or $m\left(U_{1} \cap U_{2}\right)=m\left(U_{2}\right)$. If $U$ is an atom note that $m(E \cap U)=m(U)$ or $m(E \cap U)=0$ for any measurable set $E$. If $f \in L$, then $f$ is constant almost everywhere on $U$; we denote this constant $f(U)$. If $E$ is a measurable set for which $m(E \cap U)=0$ for every atom $U$, we say that $E$ is nonatomic. In particular, if there are no atoms in $X$ we say that $(X, m)$ is a nonatomic measure space.

THEOREM. Let $V$ be a vector subspace of $L$ under the usual operations of scalar multiplication and addition of vectors which contains all the simple functions on $X$. Let $V$ be endowed with a Hausdorff topology for which $V$ is a topological vector space such that there exists a nondecreasing function $\phi$ mapping the nonnegative real axis into itself so that a complete neighborhood system of $\theta$ in $V$ is given by sets of the form $\{f \in V ; \phi(|f|)<\varepsilon\}$. Then

(i) if $\lim \inf n^{-1} \phi(n)>0$, then given any two linearly independent functions $f$ and $g$ in $V$ there is a continuous linear functional $T$ on $V$ for which $T f \neq 0$ and $T g=0$.

(ii) if $\lim \inf n^{-1} \phi(n)=0$ and $\left\{U_{\alpha}\right\}$ is the (possibly vacuous) family of all atoms in $X$, then given any continuous linear functional $T$ on $V$ we have $T f=\Sigma_{\alpha} f\left(U_{\alpha}\right) T\left(\chi U_{\alpha}\right)$ for all $f \in V$; in particular, if $(X, m)$ is nonatomic there are no nonzero continuous linear functionals on $V$.

Since $\lim \inf n^{-1} \phi(n) \geqq 0$, statements (i) and (ii) exhaust all possibilities. Before developing a proof we note that the Banach space $L_{p}(X, m)$ and the examples of Banach and Day are all special cases of our Theorem. In Banach's example $\phi(x)=x(1+x)^{-1}$ (we can show that $\phi$ is nondecreasing by differentiating $\phi$ ) and plainly case (ii) applies. In the Banach space $L_{p}(X, m)$ and in Day's example $\phi(x)=$ $x^{p}$; in the former problem case (i) applies and in the latter, case (ii) applies. (Completeness is employed in the proof of Day's example in [3]; however completeness will not enter into the argument presented here.)

Proof. We can assume without loss of generality that $V \neq(0)$, i.e., there is a function $f$ in $V$ for which $f \neq \theta$. We claim that there 
is a measurable set $E$ for which $0<m(E)<\infty$. To prove this observe that there is an integer $n$ so large that $\int \phi\left(\left|n^{-1} f\right|\right)<1$. Since $V$ is Hausdorff $\int \phi\left(\left|n^{-1} f\right|\right)>0$ and there must be a real number $c>0$ for which $0<m X\left(\phi\left(\left|n^{-1} f\right|\right) \geqq c\right)<\infty$.

We claim that $\phi(0)=0$ and $\lim _{y \rightarrow 0^{+}} \phi(y)=0$. To see this observe that $\theta$ is in every neighborhood of itself; consequently $0=\int \dot{\phi}(\theta)$ and $\phi(0)=0$. Let $E$ be a measurable set for which $0<m(E)<\infty$. Because $V$ is a topological vector space $n^{-1} \chi_{E}$ converges in $V$ to $\theta$ as $n \rightarrow \infty$ and

$$
\int \phi\left(n^{-1} \chi_{E}\right)=m(E) \phi\left(n^{-1}\right) \rightarrow 0 \quad \text { as } n \rightarrow \infty .
$$

Since $\phi$ is nondecreasing we have $\lim _{y \rightarrow 0^{+}} \phi(y)=0$.

We claim that $y \geqq 0$ and $\phi(y)=0$ imply $y=0$. For if $y>0$ then (because $V$ is Hausdorff) $m(E) \phi(y)=\int \phi\left(y \chi_{E}\right)>0$ and $\phi(y)>0$ where $E$ is a set for which $0<m(E)<\infty$.

We claim that given any $\varepsilon>0$ there is a $\delta>0$ such that $y \geqq 0$ and $\phi(y)<\delta$ imply $y<\varepsilon$. If this were not true there would be a sequence of numbers $y_{n} \geqq \varepsilon$ for which $\lim _{n \rightarrow \infty} \phi\left(y_{n}\right)=0$; but then $\phi(\varepsilon)=0$ which is impossible.

Let $V^{\prime}$ be the vector subspace of $V$ composed of all the simple functions. We claim that $V^{\prime}$ is dense in $V$. To prove this let $f$ be an arbitrary function in $V$. There is an integer $n$ such that $\int \varphi\left(\left|n^{-1} f\right|\right)<1$. On any set of the form $X\left(K_{1} \geqq|f| \geqq K_{2}>0\right) f$ can be uniformly approximated by functions in $V^{\prime}$ and it follows from the Dominated Convergence Theorem that $n^{-1} f$ and $f$ are in the closure of $V^{\prime}$ in $V$ (recall that $\lim _{y \rightarrow 0^{+}} \phi(y)=0$ ).

To prove (i) suppose that lim inf $n^{-1} \phi(n)>0$ and let $f, g$ be linearly independent functions in $V$. Hence there is a number $c>0$ for which $f \chi_{E}$ and $g \chi_{E}$ are linearly independent where $E=X(|f| \geqq c) \cup X(|g| \geqq c)$; otherwise there would exist scalars $a, b$ for which $a^{2}+b^{2}>0$ and $a f \chi_{E}+b g \chi_{E}=0$ almost everywhere for all $c>0$, and hence $a f+$ $b g=0$ almost everywhere on $X$ which is impossible. Note that $0<$ $m(E)<\infty$.

We have lim $\inf _{y \rightarrow \infty} y^{-1} \phi(y)=\lim \inf n^{-1} \phi(n)$ because $\phi$ is nondecreasing. Then there are positive numbers $\delta$ and $K$ such that if $y>K$, then $y^{-1} \phi(y) \geqq \delta$ and $\delta^{-1} \phi(y) \geqq y$. There is an integer $n$ so large that $\int \phi\left(\left|n^{-1} f\right|\right)<1$. For $n^{-1}|f(x)|>K$ it follows that

$$
|f(x)| \leqq n \delta^{-1} \phi\left(\left|n^{-1} f(x)\right|\right)
$$

and consequently $f \chi_{E}$ is in $L_{1}(E, m)$ where $E$ is the set given in the 
preceding paragraph. Likewise $h \chi_{E}$ is in $L_{1}(E, m)$ for any $h$ in $V$.

But $L_{1}(E, m)$ is a Banach space and by the Hahn-Banach Theorem there is a continuous linear functional $S$ on $L_{1}(E, m)$ for which $S\left(f \chi_{E}\right) \neq 0$ and $S\left(g \chi_{E}\right)=0$. Define the linear functional $T$ on $V$ as. follows; $T h=S\left(h \chi_{E}\right)$ for all $h$ in $V$.

To complete the proof of (i) it suffices to show that $T$ is continuous on $V$. Now $V$ is evidently first countable. We need only show that if $\left\{h_{n}\right\}$ is a sequence of functions in $V$ converging to $\theta$ in $V$, then $\left\{h_{n} \chi_{E}\right\}$ converges to $\theta$ in $L_{1}(E, m)$. Let $\left\{h_{n}\right\}$ be such a sequence; then $\lim \int \phi\left(\left|h_{n}\right|\right)=0$ and $\phi\left(\left|h_{n}\right|\right)$ converges to $\theta$ in measure on $X$. But given any $\varepsilon>0$ there is a $\delta>0$ such that $y \geqq 0$ and $\phi(y)<\delta$ imply $y<\varepsilon$. Hence $\left\{h_{n}\right\}$ converges to $\theta$ in measure on $X$. But by an argument above

$$
\left|h_{n}(x)\right| \leqq \delta^{-1} \phi\left(\left|h_{n}(x)\right|\right)
$$

if $\left|h_{n}(x)\right|>K$ for certain fixed numbers $\delta$ and $K$; it follows that $\lim _{n \rightarrow \infty} \int_{E}\left|h_{n} \chi_{E}\right|=0$ and (i) is proved.

To prove (ii) suppose $\lim \inf n^{-1} \phi(n)=0$ and let $T$ be a continuous linear functional on $V$. We claim that if $E$ is a nonatomic measurable set for which $0<m(E)<\infty$, then $T \chi_{E}=0$. To prove this suppose $T \chi_{E} \neq 0$ and select any integer $n>0$. It is well known that the set $\{m(S) ; S \subset E\}$ is the closed interval [0,m(E)] (see [4, pp. 308-9] for a proof that this set is dense in the interval, and the rest of the argument is routine). By induction there exists mutually disjoint subsets $E_{1}, E_{2}, \cdots, E_{n}$ of $E$ for which $m\left(E_{i}\right)=n^{-1} m(E)$ for all $i$. Then

$$
\left|T \chi_{E}\right|=\left|\sum_{1}^{n} T \chi_{E_{i}}\right| \leqq \sum_{1}^{n}\left|T \chi_{E_{i}}\right|
$$

and plainly there exists an index $j$ for which $\left|T \chi_{E}\right| \geqq n^{-1}\left|T \chi_{E}\right|$. Hence

$$
\int_{X} \phi\left(n \chi_{E_{j}}\right)=\int_{E} \phi(n)=\phi(n) m\left(E_{j}\right)=n^{-1} \phi(n) m(E) ;
$$

but on the other hand

$$
\left|T\left(n \chi_{E_{j}}\right)\right|=n\left|T \chi_{E_{j}}\right| \geqq n n^{-1}\left|T \chi_{E}\right|=\left|T \chi_{E}\right|>0 .
$$

Because liminf $n^{-1} \phi(n)=0$ it follows that in every neighborhood of $\theta^{\circ}$ in $V$ there is a function $f$ for which $|T f| \geqq\left|T \chi_{E}\right|$ contrary to the hypothesis that $T$ is continuous.

Observe that $T f=0$ for any simple function $f$ with nonatomic support.

To conclude the proof of (ii) let $f$ be any function in $V$ for which 
$\int \phi(|f|)<1$. There is a sequence of simple functions $\left\{h_{n}\right\}$ such that $\lim \int \phi\left(\left|f-h_{n}\right|\right)=0$. Put $E=X-\mathrm{U}_{a} U_{\alpha}$. Now $f\left(U_{\alpha}\right) \neq 0$ for at most countably many $U_{\alpha}$; say $U_{1}, U_{2}, U_{3}, \cdots$ are these atoms enumerated. Clearly $f \chi_{E}$ and $h_{n} \chi_{E}$ are measurable and $\lim \int \phi\left(\left|f \chi_{E}-h_{n} \chi_{E}\right|\right)=0$. Put $g_{n}=h_{n} \chi_{E}+\sum_{1}^{n} f\left(U_{i}\right) \chi_{\sigma_{i}}$. Now $\sum_{1}^{\infty} \phi\left[\left|f\left(U_{i}\right)\right|\right] m\left(U_{i}\right) \leqq \int \phi(|f|)<\infty$. Consequently

$$
\int \phi\left(\left|f-g_{n}\right|\right)=\int \phi\left(\left|f \chi_{E}-h_{n} \chi_{E}\right|\right)+\sum_{n+1}^{\infty} \phi\left[\left|f\left(U_{i}\right)\right|\right] m\left(U_{i}\right)
$$

and $g_{n}$ converges to $f$ in $V$. But

$$
T g_{n}=T\left(h_{n} \chi_{E}\right)+\sum_{1}^{n} f\left(U_{i}\right) T\left(\chi_{\sigma_{i}}\right)=\sum_{1}^{n} f\left(U_{i}\right) T\left(\chi_{\sigma_{i}}\right) .
$$

Because $T$ is continuous

$$
T f=\sum_{1}^{\infty} f\left(U_{i}\right) T\left(\chi_{\sigma_{i}}\right)=\sum_{\alpha} f\left(U_{\alpha}\right) T\left(\chi_{\sigma_{\alpha}}\right) .
$$

This holds for any function $f$ in $V$ (because we can always consider $n^{-1} f$ where $\left.\int \phi\left(\left|n^{-1} f\right|\right)<1\right)$. The proof of (ii) is complete.

In our Theorem (ii) suggests the possibility of constructing several topological vector spaces which have no nonzero continuous linear functionals. We present one explicitly by employing the following

INEQUALITY. For any nonnegative numbers $x$ and $y$

$$
\frac{x}{\log (9+x)}+\frac{y}{\log (9+y)} \geqq \frac{x+y}{\log (9+x+y)}
$$

and equality holds if and only if $x=0$ or $y=0$.

Proof. Set $\phi(x)=x / \log (9+x)$ and observe that

$$
\phi^{\prime \prime}(x)=\frac{-18 \log (9+x)-x[\log (9+x)-2]}{(9+x)^{2} \log ^{3}(9+x)}
$$

and $\phi^{\prime \prime}(x)<0$ for all $x \geqq 0$ because $9>e^{2}$. We can suppose without loss of generality that $x \geqq y>0$. Then $\phi^{\prime}(x)$ is strongly decreasing and by the Mean Value Theorem

$$
\phi(x+y)-\phi(x)<\phi(y)-\phi(0)
$$

and the conclusion follows. Observe also that $\phi^{\prime}(x) \geqq 0$ and $\phi$ is strongly increasing for $x \geqq 0$.

To construct our example let $(X, m)$ be a nonatomic measure space 
and let $V$ be the subset of $L$ composed of all functions $f$ for which $\int \phi(|f|)<\infty$ where $\phi(y)=y[\log (9+y)]^{-1}$. That $V$ is closed under addition follows from the Inequality. To show that $V$ is closed under scalar multiplication observe that

$$
|c f|[\log (9+|c f|)]^{-1} \leqq|f|[\log (9+|f|)]^{-1}
$$

if $|c| \leqq 1$ and

$$
|c f|[\log (9+|c f|)]^{-1} \leqq|c||f|[\log (9+|f|)]^{-1}
$$

if $|c|>1$. And in either event

$$
|c f|[\log (9+|c f|)]^{-1} \leqq(1+|c|)|f|[\log (9+|f|)]^{-1} \text {. }
$$

Thus $V$ is a vector subspace of $L$ under the usual operations of addition and scalar multiplication of vectors. Define a metric $\rho$ on $V$ as follows; $\rho(f, g)=\int \phi(|f-g|)$. That $\rho$ is a metric follows from our Inequality and trivial verifications.

We claim that $V$ is a topological vector space under the metric topology. That $(f, g) \rightarrow f+g$ is continuous in $f$ and $g$ simultaneously follows from the Inequality. To show that $(c, f) \rightarrow c f$ is continuous in $c$ and $f$ simultaneously we observe that

$$
\begin{aligned}
\rho(c f, & \left.c_{0} f_{0}\right) \\
& =\int\left|c f-c_{0} f_{0}\right|\left[\log \left(9+\left|c f-c_{0} f_{0}\right|\right)\right]^{-1} \\
& \leqq \int\left|c f-c f_{0}\right|\left[\log \left(9+\left|c f-c f_{0}\right|\right)\right]^{-1} \\
& +\int\left|c f_{0}-c_{0} f_{0}\right|\left[\log \left(9+\left|c f_{0}-c_{0} f_{0}\right|\right)\right]^{-1} \\
& \leqq(1+|c|) \int\left|f-f_{0}\right|\left[\log \left(9+\left|f-f_{0}\right|\right)\right]^{-1} \\
& +\int\left|c-c_{0}\right|\left|f_{0}\right|\left[\log \left(9+\left|c-c_{0}\right|\left|f_{0}\right|\right)\right]^{-1} .
\end{aligned}
$$

In the preceding paragraph fix $c_{0}$ and $f_{0}$ and let $c$ and $f$ vary. Now if $\left|c-c_{0}\right|<1$ then

$$
\left|c-c_{0}\right|\left|f_{0}\right|\left[\log \left(9+\left|c-c_{0}\right|\left|f_{0}\right|\right)\right]^{-1} \leqq\left|f_{0}\right|\left[\log \left(9+\left|f_{0}\right|\right)\right]^{-1} .
$$

By the Dominated Convergence Theorem we can make $\rho\left(c f, c_{0} f_{0}\right)$ as small as we please by making $\left|c-c_{0}\right|$ and $\rho\left(f, f_{0}\right)$ small enough. Hence $V$ is a topological vector space satisfying (ii) in our Theorem and there is no nonzero continuous linear functional on $V$. (By an argument paralleling the proof that $L_{p}(X, m)$ is complete for $p \geqq 1$ one can also show that $V$ is complete; the details are left to the 
reader.)

The interested reader can also show that the set of functions $f$ in $L$ for which $\int \log (1+|f|)<\infty$ endowed with the metric $\rho(f, g)=$ $\int \log (1+|f-g|)$ is a topological vector space under the metric topology which has no nonzero continuous linear functional.

\section{REFERENCES}

1. S. Banach, Theorie Les Operations Lineares, Chelsea, 1955.

2. S. Cater Note on a theorem of Day, Amer. Math. Monthly 69 (1962), 638-640.

3. M. M. Day. The Spaces $L^{p}$ With $0<p<1$, Bull. Amer. Math. Soc. 46 (1940), 816-823.

4. N. Dunford and J. T. Schwartz, Linear operators, Part I Interscience, 1958.

5. P. R. Halmos, Measure Theory, van Nostrand, 1950.

UNIVERSITY OF OREGON 



\section{PACIFIC JOURNAL OF MATHEMATICS}

\section{EDITORS}

\section{RalPh S. Phillips}

Stanford University

Stanford, California

M. G. Arsove

University of Washington

Seattle 5, Washington
J. Dugundji

University of Southern California Los Angeles 7, California

Lowell J. Paige

University of California

Los Angeles 24, California

\section{ASSOCIATE EDITORS}
E. F. BECKENBACH
D. DERRY
H. L. ROYDEN
E. G. STRAUS
T. M. CHERRY
M. OHTSUKA
E. SPANIER
F. WOLF

\section{SUPPORTING INSTITUTIONS}

\author{
UNIVERSITY OF BRITISH COLUMBIA \\ CALIFORNIA INSTITUTE OF TECHNOLOGY \\ UNIVERSITY OF CALIFORNIA \\ MONTANA STATE UNIVERSITY \\ UNIVERSITY OF NEVADA \\ NEW MEXICO STATE UNIVERSITY \\ OREGON STATE UNIVERSITY \\ UNIVERSITY OF OREGON \\ OSAKA UNIVERSITY \\ UNIVERSITY OF SOUTHERN CALIFORNIA
}

STANFORD UNIVERSITY

UNIVERSITY OF TOKYO

UNIVERSITY OF UTAH

WASHINGTON STATE UNIVERSITY

UNIVERSITY OF WASHINGTON

AMERICAN MATHEMATICAL SOCIETY

CALIFORNIA RESEARCH CORPORATION SPACE TECHNOLOGY LABORATORIES

NAVAL ORDNANCE TEST STATION

Printed in Japan by International Academic Printing Co., Ltd., Tokyo Japan 


\section{Pacific Journal of Mathematics}

\section{Vol. 13, No. 1 \\ March, 1963}

Frantz Woodrow Ashley, Jr., A cone of super-(L) functions............. 1

Earl Robert Berkson, Some metrics on the subspaces of a Banach space....

Felix Earl Browder and Walter Strauss, Scattering for non-linear wave

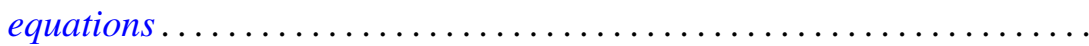

Edmond Darrell Cashwell and C. J. Everett, Formal power series ..........

Frank Sydney Cater, Continuous linear functionals on certain topological

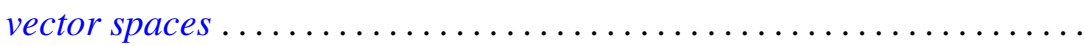

John Douglas Dixon, General group extensions ....................

Robert Pertsch Gilbert, On harmonic functions of four variables with

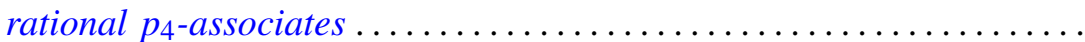

Irving Leonard Glicksberg, On convex hulls of translates ..............

Simon Hellerstein, On a class of meromorphic functions with deficient zeros

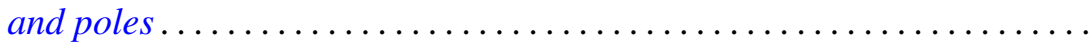

Donald William Kahn, Secondary cohomology operations which extend the

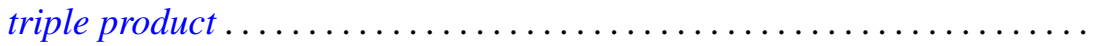

G. K. Leaf, A spectral theory for a class of linear operators .............

R. Sherman Lehman, Algebraic properties of the composition of solutions of partial differential equations ........................... 157

Joseph Lehner, On the generation of discontinuous groups ............. 169

S. P. Lloyd, On certain projections in spaces of continuous functions ...... 171 Fumi-Yuki Maeda, Generalized spectral operators on locally convex spaces ..................................

Donald Vern Meyer, $E^{3}$ modulo a 3-cell

William H. Mills, An application of linear programming to permutation groups.

Richard Scott Pierce, Centers of purity in abelian groups

Christian Pommerenke, On meromorphic starlike functions ...

Zalman Rubinstein, Analytic methods in the study of zeros of

polynomials...

B. N. Sahney, On the Nörlund summability of Fourier series

Tôru Saitô, Regular elements in an ordered semigroup . .

Lee Meyers Sonneborn, Level sets on spheres...........

Charles Andrew Swanson, Asymptotic estimates for limit point

problems .

Lucien Waelbroeck, On the analytic spectrum of Arens . .

Alvin (Murray) White, Singularities of a harmonic function of three

variables given by its series development .............

Kōichi Yamamoto, Decomposition fields of difference sets ...

Chung-Tao Yang, On the action of $\mathrm{SO}(3)$ on a cohomology manifold... 